\title{
A generalized advection dispersion equation
}

\author{
Abdon Atangana \\ Institute for Groundwater Studies, Faculty of Natural and Agricultural Sciences, \\ University of the Free State, Bloemfontein 9300, South Africa. \\ e-mail: abdonatangana@yahoo.fr
}

This paper examines a possible effect of uncertainties, variability or heterogeneity of any dynamic system when being included in its evolution rule; the notion is illustrated with the advection dispersion equation, which describes the groundwater pollution model. An uncertain derivative is defined; some properties of the operator are presented. The operator is used to generalize the advection dispersion equation. The generalized equation differs from the standard equation in four properties. The generalized equation is solved via the variational iteration technique. Some illustrative figures are presented.

\section{Introduction}

The distribution of sedimentary facies controls the heterogeneity of hydrogeological properties of porous sedimentary aquifers at different scales. The arrangement of individual facies and their porosity and permeability determine the path of groundwater flow across sedimentary bodies. Therefore the capability to forecast hydrogeological heterogeneity due to facies changes helps to improve solutions of flow and diffusion problems in this kind of aquifer. When real aquifers are studied, it is impossible to model groundwater flow at a scale such that we can take into account the effects of fine-scale sedimentary heterogeneity; in fact this would require a precise knowledge of the sedimentary bodies that cannot be obtained from sparse data at some wells and this would be prohibitive for the required computing power. Therefore the fine scale heterogeneity is usually 'up-scaled' and the heterogeneous real medium is substituted at a larger scale with an equivalent often anisotropic medium, whose parameters allow the reproduction of the average flow of the real heterogeneous sedimentary structure. It is very important to notice that there is a close relationship between the flow and the transport of the pollution through the geological formation. The description of transport is closely related to the terms convection, diffusion, dispersion, and retardation as well as decomposition. First, it is assumed that there are no interactions between the species dissolved in water and the surrounding solid phase (Javandel et al. 1984). The primary mechanism for the transport of improperly discarded hazardous waste through the environment is by the movement of water through the subsurface and surface waterways. Study of this movement requires that one must be able to measure the quantity of waste present at a particular point in space and time. The measure, universally, for chemical pollution is the concentration. Analytical methods that handle solute transport in porous media are relatively easy to use (Javandel et al. 1984). However, because of complexity of the equations involved, the analytical solutions generally available are restricted to either radial flow problems or to cases where velocity is uniform over the area of interest or the area under investigation is homogeneous. Neglecting such aspects in groundwater assessments can lead

Keywords. Uncertain derivative; groundwater pollution model; variational iteration method. 
to incorrect results and misleading output. Up to now the notion of variability or nonhomogeneity in groundwater assessment is not well known. It is not easy to precisely determine how the aquifer system varies from one point to another and how it will vary as time goes. This notion of heterogeneity can then be classified under uncertainties in groundwater assessment. Generally there are other various sources of uncertainty in model outputs, for example, uncertainty associated with lack of knowledge or accuracy of the model inputs as well as the structural uncertainty related to the mathematical interpretation of the model. The assessment and presentation of the effects of uncertainty are now widely recognized as important parts of analyses for complex systems (Mandeibrot 1982; Gloecke and Nonnemacher 1993; Metzler and Klafter 2000; Yanovsky et al. 2000; Chechkin et al. 2002). At the simplest level such analyses can be viewed as the study of functions. In order to include explicitly the possible effect of the uncertainties into mathematical models, we have introduced in this paper, the uncertainties in groundwater models as a function of time and space.

$$
u=u(\underline{x}, t) .
$$

The idea of modelling the uncertainties may be generalized in all dynamic systems. A dynamical system is a concept in mathematics where a fixed rule describes the time dependence of a point in a geometrical space (Alligood et al. 2000). At any given time a dynamical system has a state given by a set of real numbers (a vector) that can be represented by a point in an appropriate state space (a geometrical manifold). Small changes in the state of the system create small changes in the numbers (Alligood et al. 2000). The evolution rule of the dynamical system is a fixed rule that describes what future states follow from the current state. The rule is deterministic; in other words, for a given time interval only one future state follows from the current state (Alligood et al. 2000). In mathematical terms, a dynamic system is a tuple $(\mathfrak{D}, f, T$ ) with $\mathfrak{D}$ a manifold (locally a Banach space or Euclidean space), $T$ the domain for time (non-negative real, the integers) and $f$ an evolution rule $t \rightarrow f^{t}$ (with $t \in T$ ) such that $f^{t}$ is a diffeomorphism of a manifold to itself (Palis and de Melo 1982).

In this paper the idea of including the uncertainties into the mathematical formulation of an evolution rule of a dynamic system will be illustrated with the advection diffusion equation (the groundwater pollution model). The paper starts with the following definition and properties.

\section{Definition and properties}

\subsection{Definition}

Let $\mathfrak{D}$ be a dynamic system with domain $T$ (domain for time), and $u \geq 0$ an uncertainty function of $\mathfrak{D}$ within $T$, then if $f \in \mathfrak{D}$, the uncertain derivative of function $f$ denoted by $D^{u} f$ is defined as:

$$
D^{u} f(x)=(1+u(x)) f^{\prime}(x)+u^{\prime}(x) f(x) .
$$

Existence of the operator

$$
\begin{aligned}
\left|D^{u} f(x)\right| & =\left|(1+u(x)) f^{\prime}(x)+u^{\prime} f(x)\right| \\
& \leq|(1+u(x))|\left|f^{\prime}(x)\right|+\left|u^{\prime}(x)\right||f(x)|
\end{aligned}
$$

therefore, if $f(x), f^{\prime}(x), u(x)$ and $u^{\prime}(x)$ exist then $D^{u} f(x)$ exists.

\subsection{Some properties of the operator (Atangana and Kiliçman 2013a)}

Let us examine some properties of the above derivative operator

- Addition

If $u_{x}, f(x)$ and $g(x)$ are differentiable in the opened interval $\mathfrak{D}$, then:

$D^{u_{x}}[f(x)+g(x)]=D^{u_{x}}[f(x)]+D^{u_{x}}[g(x)]$.

\section{Proof}

$$
\begin{aligned}
D^{u_{x}}[f(x)+g(x)]=(1 & \left.+u_{x}\right) \frac{\partial[f(x)+g(x)]}{\partial x} \\
& +\frac{\partial u_{x}}{\partial x}[f(x)+g(x)]\left(1+u_{x}\right) \\
& \times \frac{\partial[f(x)]}{\partial x}+\frac{\partial u_{x}}{\partial x}[f(x)] \\
& +\left(1+u_{x}\right) \frac{\partial[g(x)]}{\partial x} \\
& +\frac{\partial u_{x}}{\partial x}[g(x)] . \\
= & D^{u_{x}}[f(x)]+D^{u_{x}}[f(x)] .
\end{aligned}
$$

- Division

If $u_{x}$ and $1 /(f(x))$ are differentiable on the opened interval $\mathfrak{D}$, then:

$$
\begin{aligned}
D^{u_{x}}\left[\frac{1}{f(x)}\right] & =\frac{\left[-\left(1+u_{x}\right) f^{\prime}(x)+u_{x}^{\prime} f(x)\right]}{f^{2}(x)} \\
& =\frac{-f^{\prime}(x)}{f^{2}(x)}-\frac{u_{x} f^{\prime}(x)}{f^{2}(x)}+\frac{u_{x}^{\prime} f(x)}{f^{2}(x)} .
\end{aligned}
$$




\section{- Multiplication}

If $u_{x}, f(x)$ and $g(x)$ are differentiable in the opened interval $\mathfrak{D}$, then:

$$
\begin{aligned}
& D^{u_{x}}[f(x) \cdot g(x)]=g(x) f^{\prime}(x)+f(x) g^{\prime}(x) \\
& \quad+\left(g f^{\prime}+f g^{\prime}\right)(x) u_{x}+u_{x}^{\prime}(f(x) g(x)) .
\end{aligned}
$$

\section{- Power}

If $u_{x}$ and $f(x)$ are differentiable in the opened interval $\mathfrak{D}$, then

$$
\begin{aligned}
D^{1+u_{x}} & {\left[(f(x))^{n}\right] } \\
& =n f^{\prime} f^{n-1}+u_{x} n f^{\prime} f^{n-1}+u_{x}^{\prime} f^{n}, \quad n \geq 1 .
\end{aligned}
$$

- If $u_{x}$ and $f(x)$ are two times differentiable in the opened interval $\mathfrak{D}$, then:

$$
\begin{aligned}
D^{u_{x}}\left[D^{u_{x}}[f(x)]\right]= & \left(1+u_{x}\right)\left[\left(1+u_{x}\right) \frac{\partial^{2} f}{\partial x^{2}}\right. \\
& \left.+3 \frac{\partial f}{\partial x} \frac{\partial u_{x}}{\partial x}+\frac{\partial^{2} u_{x}}{\partial x^{2}} f\right]+\frac{\partial u_{x}}{\partial x} f
\end{aligned}
$$

- Clauraut's theorem for the approximation Assume that $f(x, y), u_{x}$ and $u_{y}$ are functions for which $\frac{\partial^{2} f}{\partial x \partial y}, \frac{\partial^{2} f}{\partial y \partial x}, \frac{\partial^{2} \varepsilon_{x}}{\partial x \partial y}$ and $\frac{\partial^{2} \varepsilon_{y}}{\partial x \partial y}$ exist and are continuous over a domain $\mathrm{D} \subset$ $\mathbb{R}^{2}$ then, $D^{1+u_{x}}\left[D^{1+u_{y}}[f(x, y)]\right]$ and $D^{1+u_{y}} \times$ $\left[D^{1+u_{x}}[f(x, y)]\right]$ exist and are continuous over the domain $D$. In addition, if $u_{x}=u_{y}$ then,

$$
D^{u_{y}}\left[D^{u_{x}}[f(x, y)]\right]=D^{u_{x}}\left[D^{u_{y}}[f(x, y)]\right] .
$$

Proof. If $f(x, y), u_{x}$ and $u_{y}$ are functions continuous for which $\frac{\partial^{2} f}{\partial x \partial y}, \frac{\partial^{2} f}{\partial y \partial x}, \frac{\partial^{2} \varepsilon_{x}}{\partial x \partial y}$ and $\frac{\partial^{2} \varepsilon_{y}}{\partial x \partial y}$ exist and are continuous over a domain an open domain $D \subset \mathbb{R}^{2}$ then,

$$
\begin{aligned}
D^{u_{y}}\left[D^{u_{x}}[f(x, y)]\right] & \\
=\left(1+u_{y}\right) & {\left[\frac{\partial u_{x}}{\partial y} \frac{\partial f}{\partial x}+\frac{\partial^{2} f}{\partial y \partial x}+\frac{\partial^{2} u_{x}}{\partial y \partial x} f(x, y)\right.} \\
& \left.+\frac{\partial u_{x}}{\partial x} \frac{\partial f}{\partial y}\right] \\
& +\left(1+u_{x}\right) \frac{\partial u_{y}}{\partial y} \frac{\partial f}{\partial x}+\frac{\partial u_{y}}{\partial y} \frac{\partial u_{x}}{\partial x} f(x, y)
\end{aligned}
$$

Then, $D^{u_{y}}\left[D^{u_{x}}[f(x, y)]\right]$ expression exists as sum of existing function. Now interchanging $x$ by $y$ we obtain the same result with $D^{u_{x}}\left[D^{u_{y}}[f(x, y)]\right]$.

If $u_{x}=u_{y}$, then $\frac{\partial^{2} f}{\partial y \partial x}=\frac{\partial^{2} f}{\partial x \partial y}$ according to Clauraut's theorem, thus replacing $\frac{\partial^{2} f}{\partial x \partial y}$ by $\frac{\partial^{2} f}{\partial y \partial x}$ in $D^{u_{y}}\left[D^{u_{x}}[f(x, y)]\right]$, we obtain

$$
D^{u_{y}}\left[D^{u_{x}}[f(x, y)]\right]=D^{u_{x}}\left[D^{u_{y}}[f(x, y)]\right] .
$$

- Chain-rule for the approximation

$$
\begin{aligned}
& D^{u_{x}}(f \circ g)=\left(1+u_{x}\right) g^{\prime}(x) f^{\prime}[g(x)]+\frac{\partial u_{x}}{\partial x}(f \circ g) \\
& =g^{\prime}(x) f^{\prime}[g(x)]+u_{x} g^{\prime}(x) f^{\prime}[g(x)]+\frac{\partial u_{x}}{\partial x}(f \circ g) .
\end{aligned}
$$

- Rolle's theorem for the approximation

If a real-valued function $f$ and $u_{x}$ are continuous functions on a closed interval $(a, b)$, differentiable on the open interval $(a, b)$, and $f(a)=f(b)$, then there exist a $c$ in the open interval $(a, b)$ and a small parameter $\mu$ such that

$$
D^{u_{x}} f(c)=\mu f(c) .
$$

Proof. Following Rolle's theorem, there exists a $c$ in the open interval $(a, b)$ such that $f^{\prime}(c)=0$. For this $c$ we have

$$
\begin{aligned}
D^{u_{x}} f(c) & =\left(1+u_{x}(c)\right) f^{\prime}(c)+u_{x}^{\prime}(c) f(c) \\
& =u_{x}^{\prime}(c) f(c)=\mu f(c) .
\end{aligned}
$$

- If $g(x), f(x)$ and $u_{x}$ are differentiable in $\mathfrak{D}$, then there exist $\alpha>1$ and $\beta>0$ such that:

$$
\begin{aligned}
& \left|D^{u_{x}} f(x)-D^{u_{x}} g(x)\right| \leq \alpha\left|f^{\prime}(x)-g^{\prime}(x)\right| \\
& +\beta|f(x)-g(x)|, \quad \forall x \in I .
\end{aligned}
$$

Proof. Let $x \in \mathfrak{D}$, then

$$
\begin{aligned}
\left|D^{u_{x}} f(x)-D^{u_{x}} g(x)\right|= & \mid\left(1+u_{x}(x)\right) f^{\prime}(x)+u_{x}^{\prime}(x) f(x) \\
& -\left(1+u_{x}(x)\right) g^{\prime}(x)-u_{x}^{\prime}(x) g(x) \mid \\
= & \left|1+u_{x}(x)\right|\left|f^{\prime}(x)-g^{\prime}(x)\right| \\
& +\left|u_{x}^{\prime}(x)\right||f(x)-g(x)|
\end{aligned}
$$

but $u_{x}(x)$ is very small such that $\left|1+u_{x}(x)\right|<2$ and $\left|u_{x}^{\prime}(x)\right|>0$, it follows that

$$
\begin{gathered}
\left|1+u_{x}(x)\right|\left|f^{\prime}(x)-g^{\prime}(x)\right|+\left|u_{x}^{\prime}(x)\right||f(x)-g(x)| \\
\leq \alpha\left|f^{\prime}(x)-g^{\prime}(x)\right|+\beta|f(x)-g(x)| .
\end{gathered}
$$


It is important to observe that if $u=0$, we recover the properties of normal derivatives.

\section{Application on the advection dispersion equation}

The concern here is the modification of advection dispersion equation by including a possible effect of heterogeneity or variability of the aquifer into the mathematical formulation. A one dimensional model consisting of an infinitely long homogeneous isotropic porous medium with a steady state uniform flow with a seepage velocity $v$ is considered here. A particular chemical from one end of the model for a period of time $t_{0}$ such that the input concentration varies as an exponential function of time. The value of that chemical concentration at any time $t$ and at a distance $x$ from the injection boundary, allowing for the decay and adsorption, may be obtained from the solution of the following set of equations (Cleary and Ungs 1978); more details for this model can be found in (Van Genuchten and Alves 1982; Jaiswal et al. 2009, 2011; Yadav et al. 2011). Other results for this problem can be found in (Benson et al. 2000; Meerschaert et al. 2006; Zhang et al. 2007; Atangana and Kiliçman 2013b).

$$
D \frac{\partial^{2} C}{\partial x^{2}}-v \frac{\partial C}{\partial x}-\lambda R C=R \frac{\partial C}{\partial t}+f(x, t) .
$$

Subject to the initial and boundary conditions:

$$
C(x, 0)=0, \quad C(0, t)=c_{0} \exp (-\alpha t)
$$

and

$$
C_{x}(\infty, t)=0
$$

where $D$ is the dispersion coefficient, $v$ is the seepage velocity, $R$ is the retardation factor, $\lambda$ is the radioactive decay constant, $c_{0}$ is the initial concentration, $\alpha$ is a positive constant and $f(x, t)$ is any source and sink in the system. However, in the case of the groundwater pollution the function $f(x, t)$ is always neglected because we assume, there is no source and sink in the system under investigation (Atangana and Kiliçman 2013b). Therefore in our case we will set the function to be zero.

The above equation does not consider the effect of heterogeneity, variability or uncertainties in these aquifers. Therefore in order to include the effect of uncertainties, heterogeneity or variability of the geological formation into the mathematical expression, the standard derivative is replaced by the uncertainty function equation (2.1) in equation (3.1) to obtain:

$$
\begin{aligned}
& D D^{u_{x}}\left[D^{u_{x}}[C(x, t)]\right]-v D^{u_{x}}[C(x, t)]-\lambda R C(x, t) \\
& \quad=R D^{u_{t}}[C(x, t)] \\
& D\left\{\left(1+u_{x}\right)\left[\left(1+u_{x}\right) \frac{\partial^{2} C}{\partial x^{2}}+3 \frac{\partial C}{\partial x} \frac{\partial u_{x}}{\partial x}+\frac{\partial^{2} u_{x}}{\partial x^{2}} C\right]\right. \\
& \left.\quad+\frac{\partial u_{x}}{\partial x} C\right\}-v\left[\left(1+u_{x}\right) \frac{\partial c}{\partial x}+\frac{\partial u_{x}}{\partial x} C\right] \\
& \quad-\lambda R C(x, t)=R\left[\left(1+u_{t}\right) \frac{\partial c}{\partial t}+\frac{\partial u_{t}}{\partial_{t}} C\right] .
\end{aligned}
$$

The above equation is the generalized hydrodynamic advection dispersion equation. This equation takes into account the value of that chemical concentration at any time $t$ and at a distance $x$ from the injection boundary, allowing for the decay and adsorption and also the possible effect of heterogeneity, or uncertainties, variability's of the geological formation system in which the value of chemical concentration is measured.

\section{Analysis and possible solutions}

Let us put equation (3.2) in the suitable form that can easily be used for possible analytical solutions.

Suppose that the variability or uncertainties or heterogeneity of the aquifer with respect to time is very small such that addition to unity is small, then, (3.2) can be divided on both sides by $\left(1+u_{t}\right)$, in that case equation (3.2) can be roughly approximated to:

$$
\begin{aligned}
& D(x, t) \frac{\partial^{2} c}{\partial x^{2}}-v(x, t) \frac{\partial c}{\partial x}-\lambda R C+F(x) C \\
&+B(x, t) C=R \frac{\partial c}{\partial t} \\
& F(x)=D \frac{\partial^{2} u_{x}}{\partial x^{2}}-v \frac{\partial u_{x}}{\partial x} \\
& B(x, t)=\frac{\partial u_{x}}{\partial x}-R \frac{\partial u_{t}}{\partial t} \\
& D(x, t)=\left(1+u_{x}-u_{t}\right) D \\
& v(x, t)=\left(1+u_{x}-u_{t}\right) v .
\end{aligned}
$$

\subsection{Analysis}

$F(x)$ can be viewed as force of uncertainties, heterogeneity or variability of the geological formation system at each position, that acts on the constant dispersion coefficient and seepage velocity, and proportional to the value of that chemical concentration. 
$B(x, t)$ can be viewed as the proportion that allows the value of that chemical concentration to remember its trajectory in the geological formation system and the time where it was retarded since its departure from the point of injection.

$D(x, t)$ is obviously the dispersion function in each point and time in the geological formation system.

$v(x, t)$ is the seepage velocity function in each point and time in the geological formation system.

The modified equation differs from the standard version in four properties: First, the dispersion coefficient depends on time and coordinates due to the effect of uncertainties, or variability or heterogeneity of the geological formation in which the chemical is being dispersed with memory depending on time and coordinates. Secondly, seepage velocity coefficient depends on time and coordinates due to the effect of uncertainties, or variability or heterogeneity of the geological formation in which the chemical is being transported with memory depending on time and coordinates. Thirdly, the force of uncertainties, heterogeneity or variability of the geological formation system at each position, that acts on the constant dispersion coefficient and seepage velocity, and proportional to the value of that chemical concentration. Finally, we have the functional proportion, which allows, the value of that chemical concentration to remember its trajectory in the geological formation system. It also remembers the time where it was retarded since its departure from the point of injection.

\subsection{Possible analytical solution}

To solve equation (4.1) some approximation must be considered on one hand, an appropriate method of solving non-linear partial differential equation must be chosen. As V M Alexandrov wrote in the foreword of a popular science book 'Asymtotology: ideas, methods, and applications', asymptotic methods belong to the, perhaps, most romantic area of modern mathematics (Andrianov and Manevitch 2003). Though computer science is growing very fast, and numerical simulation is applied everywhere, non-numerical issues will still play a large role. Various perturbation methods have been widely applied to solve nonlinear problems. Here the variational iteration is used to find an asymptotic solution to equation (4.1).

To solve equation (4.1) the following approximations are considered. First equation (4.1) is reformulated as:

$$
D \frac{\partial^{2} C}{\partial x^{2}}-v \frac{\partial C}{\partial x}-\lambda R C-R \frac{\partial C}{\partial t}=K\left(u_{t}, u_{x} C\right) .
$$

Therefore, this equation can be solved approximately by changing the function $C$ to $C_{0}$, which satisfies equation (4.1), in terms involving $u$ (or in some of these terms). In particular, if such a change is made in all the terms, we arrive at the following hydrodynamic advection dispersion equation:

$$
\begin{aligned}
& D \frac{\partial^{2} C}{\partial x^{2}}-v \frac{\partial C}{\partial x}-\lambda R C-R \frac{\partial C}{\partial t}=K\left(u_{t}, u_{x} C_{0}\right) \\
& =K(x, t) .
\end{aligned}
$$

\subsubsection{Variational iteration method}

Variational iteration method has been favourably applied to various kinds of nonlinear problems. The main property of the method is in its flexibility and ability to solve nonlinear equations accurately and conveniently. Very recently it was recognized that the variational iteration method (He 1998a,b, 1999, 2000; Atangana 2012) and other analytical methods (Atangana et al. 2013; Atangana and Alabaraoye 2013) can be an effective procedure for solution of various nonlinear problems without usual restrictive assumptions. To solve equation (4.2) by means of variational iteration method, we put (4.2) as follows:

$D(C(x, t))_{2 x}-v(C(x, t))_{x}-\lambda R C(x, t)-K(x, t)=0$.

The correction functional for equation (4.2) can be approximately expressed as follows:

$$
\begin{aligned}
C_{n+1}(x, t)= & C_{n}(x, t)+\int_{0}^{t} \lambda_{1}(\tau)\left[D \frac{\partial^{2} \widetilde{C_{n}}}{\partial x^{2}}-v \frac{\partial \widetilde{C_{n}}}{\partial x}\right. \\
& \left.-\lambda R \check{C}_{n}-R \frac{\partial^{m} C}{\partial \tau^{m}}-\overline{K(x, \tau)}\right] d \tau,
\end{aligned}
$$

where $\lambda_{1}$ is a general Lagrange multiplier (Inokuti et al. 1978) which can be recognized optimally by means of variation assumption (Inokuti et al. 1978), here $\left(\mathrm{C}(\overline{x, \tau)})_{2 x}, \overline{K(x, \tau)}\right.$ and $\widetilde{C_{n}}(x)$ are considered as constrained variations. Making the above functional stationary, we obtain:

$$
\delta C_{n+1}(x, t)=\delta C_{n}(x, t)+\delta \int_{0}^{t} \lambda_{1}(\tau)\left[-R \frac{\partial^{m} C}{\partial \tau^{m}}\right] d \tau .
$$

Capitulating the next Lagrange multipliers, produces the following Lagrange multipliers $\lambda_{1}=-1$ for the case where $m=1$ and $\lambda_{1}=x-\zeta$ for $m=2$. For this matter $m=1$, we obtain the following iteration formula:

$$
\begin{aligned}
C_{n+1}(x, t)= & C_{n}(x, t)-\int_{0}^{t}\left[D \frac{\partial^{2} c_{n}}{\partial x^{2}}-v \frac{\partial c_{n}}{\partial x}\right. \\
& \left.-\lambda R C_{n}-R \frac{\partial^{m} C}{\partial \tau^{m}}-K(x, t)\right] d \tau .
\end{aligned}
$$


It is worth noting that if the zeroth component $C_{0}(x, t)$ is defined, then the remaining components $n \geq 1$, can be completely determined such that each term is determined by using the previous terms, and the series solutions are thus entirely determined. Finally, the solution $P(r, t)$ is approximated by the truncated series

$$
C_{N}(x, t)=\sum_{n=0}^{N-1} C_{n}(x, t)
$$

and

$$
\lim _{N \rightarrow \infty} C_{N}(r, t)=C(x, t)
$$

Example. Suppose that the average variability in space and time of the geological formation of an aquifer is governed by the following equation:

$$
u(x)=0.5 \cos (\pi x) \text { and } u(t)-1 .
$$

Following the discussion presented earlier in equation (4.2), we obtain that, the nonhomogeneous part of (4.2)

$$
\begin{aligned}
K(x, t)= & (1-0.5 \cos (\pi x)) \frac{\partial^{2} C_{0}}{\partial x^{2}} \\
& +v(0.5 \cos (\pi x)-1) \frac{\partial C_{0}}{\partial x} \\
& +\left(D \frac{\partial^{2} 0.5 \cos (\pi x)}{\partial x^{2}}-v \frac{\partial 0.5 \cos (\pi x)}{\partial x}\right) C_{0} \\
& +\frac{\partial 0.5 \cos (\pi x)}{\partial x} C_{0}
\end{aligned}
$$

here

$$
\begin{aligned}
C_{0}(x, t)= & \frac{c_{0} \exp (-\alpha t)}{2}\left[\exp \frac{x\left(q_{r}-u_{r}\right)}{2 D_{r}}\right. \\
& \quad \times \operatorname{erfc} \frac{x-u_{r} t}{2 \sqrt{D_{r} t}}+\exp \frac{x\left(q_{r}+u_{r}\right)}{2 D_{r}} \\
& \left.\times \operatorname{erfc} \frac{x+u_{r} t}{2 \sqrt{D_{r} t}}\right] \\
u_{r}= & \sqrt{q_{r}^{2}+4 D_{r}(\lambda-\alpha),} \\
& D_{r}=\frac{D}{R} \quad \text { and } \quad q_{r}=\frac{v}{R}
\end{aligned}
$$

and

$$
\operatorname{erfc}(x)=\frac{2}{\sqrt{\pi}} \int_{x}^{\infty} \exp \left(-x^{2}\right) d x
$$

is known as complementary error function and $\alpha$ is a positive constant.

Here the trajectory of the chemical concentration can be traced, but not the time of retardation.
Following the discussion presented earlier in section 4.2.1 (equation 4.3) we obtained the following recursive formula

$$
\begin{gathered}
C_{n+1}(x, t)=C_{n}(x, t)+\int_{0}^{t}\left[D \frac{\partial^{2} C_{n}}{\partial x^{2}}-v \frac{\partial C_{n}}{\partial x}\right. \\
\left.-\lambda R C_{n}-R \frac{\partial^{m} C}{\partial \tau^{m}}-K(x, t)\right] d \tau .
\end{gathered}
$$

To be simple, we chose the first component to be zero such that the second component can be determined as:

$$
C_{11}(x, t)=\int_{0}^{t} K(x, t) d \tau .
$$

In this case, two components of the decomposition series were obtained of which $P(x, t)$ was evaluated to have the following expansion:

$$
C_{1}(x, t)=C_{11}(x, t)+C_{10}(x, t)+\cdots .
$$

\section{Numerical simulation}

In this section, numerical simulations of the solution of generalized advection dispersion equation introduced in this paper together with the standard advection dispersion equation are presented. Different orders of the variable derivative are used to access the effect of the uncertainties in the mathematical formulation of the model describing the transport of solid through the geological formation called aquifer. The approximate solution of the main problem has been depicted in figure 1(a) with uncertain function $u(x, t)=1-\sin (x \cdot t)$, figure 1(b) with uncertain function $u(x, t)=1-$ $\cos (x \cdot t)$, figure $1(\mathrm{c})$ with uncertain function $u(x$, $t)=1-\sin (x)$, and the solution of the standard advection dispersion equation in figure $1(\mathrm{~d})$.

In this simulation we assume an initial concentration of $1000 \mathrm{~kg} / \mathrm{m}^{3}$ the radioactive decay to be 0.9 , the dispersion factor to be 0.1 , the velocity seepage to be $1 \mathrm{~m} /$ day and finally, we assume that the positive constant $\alpha=0.6$. It is very important to notice from figure $1(\mathrm{a}, \mathrm{b}$, and $\mathrm{c})$ that the generalized advection dispersion equation (figure $1 \mathrm{~d}$ ) is more predictable than the results presented by the advection dispersion equation. Figure 1(d) predicts that the pollution will be smooth through the aquifer while figure 1 (a, b and c) predicts that the pollution will be found in the aquifer according to the properties of the geological formation. This is mainly due to the introduction of the uncertain function representing in practise the heterogeneity of the aquifer or uncertainty in 

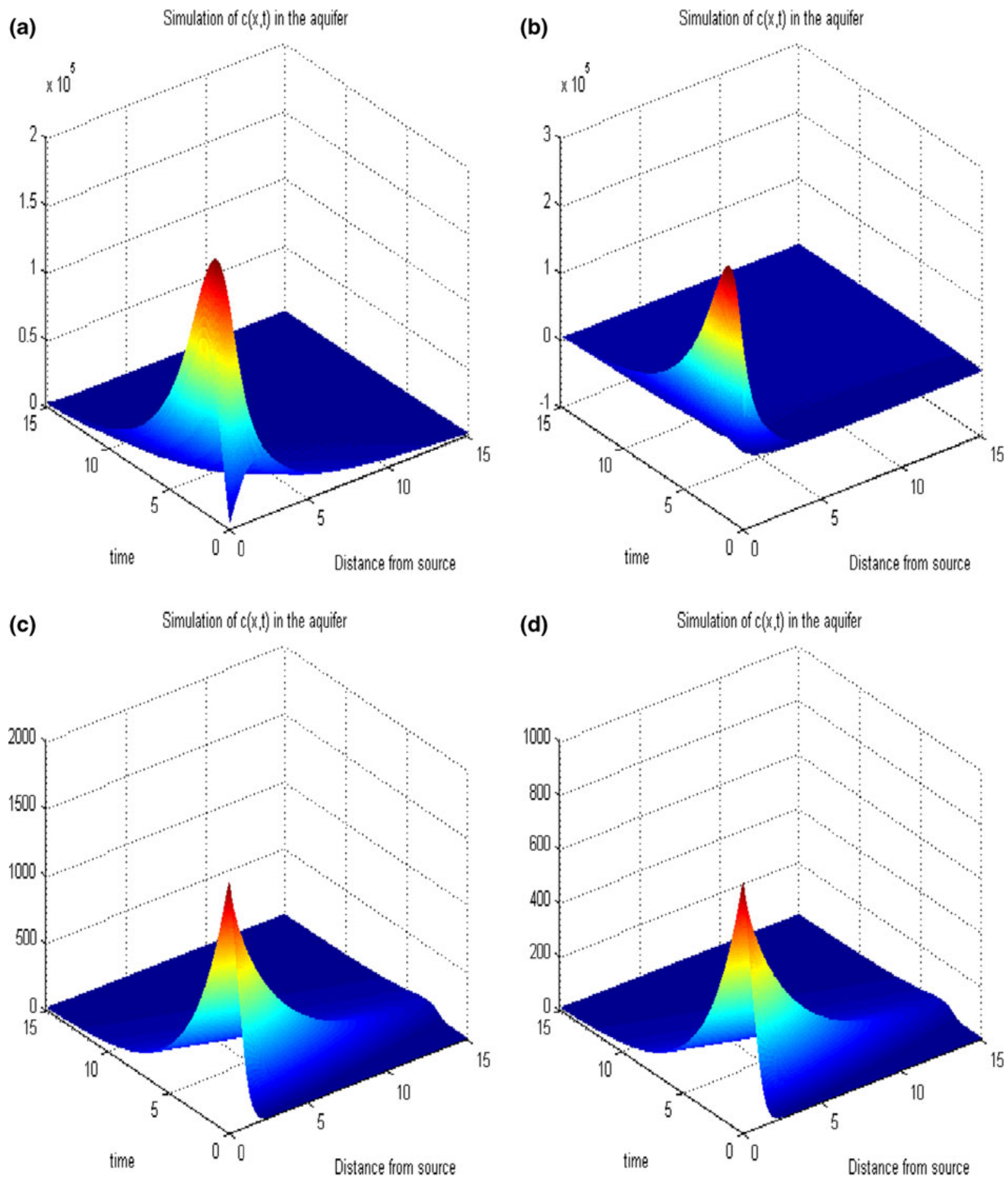

Figure 1. Numercal simulation of the approximate solution with uncertainties effect.

the geological formation through which the solutions are being transported. This is more realistic than what is predicted by the ADE.

\section{Conclusion}

The main concern in this paper was to examine the possible effects of uncertainties, variability or heterogeneity of a dynamic system when being included in its evolution rule. To achieve this, an uncertain derivative was defined and some of its properties were presented. This concept was illustrated with groundwater pollution model by using the defined operator to generalize the hydrodynamic advection dispersion equation. The generalized equation differs from the standard version in four properties: First, the dispersion coefficient depends on time and coordinates due to the effect of uncertainties, or variability or heterogeneity of 
the geological formation in which the chemical is being dispersed with memory depending on time and coordinates. Secondly, seepage velocity coefficient depends on time and coordinates due to the effect of uncertainties, or variability or heterogeneity of the geological formation in which the chemical is being transported with memory depending on time and coordinates. Thirdly, the force of uncertainties, heterogeneity or variability of the geological formation system at each position, that acts on the constant dispersion coefficient and seepage velocity, and proportional to the value of that chemical concentration. An asymptotic analytical solution is proposed via the variational iteration method.

\section{Acknowledgements}

The author would like to thank the referee for the valuable comments and helpful suggestions. A special thanks for the editor, for his valuable time spent to evaluate this manuscript.

\section{References}

Abdon Atangana 2012 New class of boundary value problems; Inf. Sci. Lett 1(2) 67-76.

Abdon Atangana and Adem Kiliçman 2013a A possible generalization of acoustic wave equation using the concept of perturbed derivative order; Mathematical Problems in Eng., vol. 2013, Article ID 696597, 6p.

Abdon Atangana and Adem Kiliçman 2013b Analytical solutions of the space-time fractional derivative of advection dispersion equation; Mathematical Problems in Eng., vol. 2013, Article ID 853127, 9p.

Abdon Atangana and Ernestine Alabaraoye 2013 Solving system of fractional partial differential equations arisen in the model of HIV infection of CD4+ cells and attractor one-dimensional Keller-Segel equation; Adv. Diff. Eq. 2013, 2013:94, doi:10.1186/1687-1847-2013-94.

Abdon Atangana, Aden Ahmed O and Necdet Bıldık 2013 A generalized version of a low velocity impact between a rigid sphere and a transversely isotropic strain-hardening plate supported by a rigid substrate using the concept of noninteger derivatives, Abstract and Appl. Analysis, vol. 2013, Article ID 671321, 9p.

Andrianov I and Manevitch L 2003 Asymptotology: Idea, Methods, and Applications; Kluwer Academic Publishers.

Benson D A, Wheatcraft S W and Meerschaert M M 2000 Application of a fractional advection-dispersion equation; Water Resour. Res. 36(6) 1403-1412.

Chechkin A V, Gorenflo R and Sokolov I M 2002 Retarding subdiffusion and accelerating superdiffusion governed by distributed-order fractional diffusion equations; Phys. Rev. B 66(4) 046129.

Cleary R W and Ungs M J 1978 Groundwater Pollution and Hydrology. Mathematical Models and Computer programs; Water Resources Programs, Princeton University, Princeton, New Jersey, 08540.

Gloecke W G and Nonnemacher T R 1993 Fox Function representation of non-Debye relaxation processes; J. Stat. Phys. 71(3/4) 741.

He J H 1998a Approximate analytical solution for seepage flow with fractional derivatives in porous media; Comput. Methods Appl. Mech. Eng. 167(1-2) 57-68.

He J H 1998b Approximate solution of nonlinear differential equations with convolution product nonlinearities; Comput. Methods Appl. Mech. Eng 167(1-2) 69-73.

He J H 1999 Variational iteration method - a kind of nonlinear analytical technique: Some examples; Int. J. Nonlinear Mech. 34(4) 699-708.

He J H 2000 Variational iteration method for autonomous ordinary differential systems; Appl. Math. Comput. 118(2-3) 115-123.

Inokuti M, Sekine H and Mura T 1978 General use of the Lagrange multiplier in non-linear mathematical physics. In: Variational method in the mechanics of solids; (ed.) Nemat-Nasser S (Oxford: Pergamon Press), pp. 156-162.

Jacob Palis and Wellington de Melo 1982 Geometric theory of dynamical systems: An introduction; Springer-Verlag, pp. 250-255.

Jaiswal D K, Kumar A, Kumar N and Yadav R R 2009 Analytical solutions for temporally and spatially dependent solute dispersion of pulse type input concentration in onedimensional semi-infinite media; J. Hydro-Environ. Res. 2 254-263.

Jaiswal D K, Kumar A, Kumar N and Singh M K 2011 Solute transport along temporally and spatially dependent flows through horizontal semi-infinite media: Dispersion being proportional to square of velocity; J. Hydrol. Eng. (ASCE) 16-3 228-238.

Javandel I, Doughty C and Tsang C F 1984 Groundwater transport: Handbook of Mathematical Models; Water Resources Monographs Series, Vol. 10, American Geophysical Union, Washington DC.

Kathleen T Alligood, Tim D Sauer and James A Yorke 2000 Chaos: An introduction to dynamical systems; SpringerVerlag, pp. 25-28.

Mandeibrot B B 1982 The Fractal Geometry of Nature; Freeman, New York.

Meerschaert M M, Mortensen J and Wheatcraft S W 2006 Fractional vector calculus for fractional advectiondispersion; Physica A 367 181-90.

Metzler R and Klafter J 2000 The random walk's guide to anomalous diffusion: A fractional dynamics approach; Phys. Rep. 3391.

Van Genuchten M Th and Alves W J 1982 Analytical solutions of one dimensional convective-dispersive solute transport equations; United State Dept. of Agriculture; Technical Bull. No. 1661.

Yadav R R, Jaiswal D K and Gulrana 2011 Two-dimensional solute transport for periodic flow in isotropic porous media: An analytical solution; Hydrol. Process. (WileyInter Science), doi: 10.1002/hyp.8398.

Yanovsky V V, Chechkin A V, Schertzer D and Tur A V 2000 Nuclear fusion driven by Coulomb explosions of large deuterium clusters; Physica A (Amsterdam) 28213.

Zhang Y, Benson D A, Meerschaert M M and LaBolle E M 2007 Space-fractional advection-dispersion equations with variable parameters: Diverse formulas, numerical solutions, and application to the MADE-site data; Water Resour. Res. 43 W05439. 